\title{
Floating drug delivery systems: A better approach
}

\author{
*Abhishek Chandel, Kapil Chauhan, Bharat Parashar, Hitesh Kumar, Sonia Arora \\ Department of Pharmacy, Manav Bharti University, Solan -173229, Himachal Pradesh, India
}

\begin{abstract}
The purpose of writing this review on floating drug delivery systems (FDDS) was to compile the recent literature with special focus on the principal mechanism of floatation to achieve gastric retention. It is known that differences in gastric physiology (such as, gastric $\mathrm{pH}$, motility) exhibit both intra- as well as inter-subject variability demonstrating significant impact on gastric retention time and drug delivery behaviour. The recent developments of FDDS including the physiological and formulation variables affecting gastric retention, approaches to design single-unit and multipleunit floating systems, and their classification and formulation aspects are covered in detail. This review also summarizes the studies to evaluate the performance and application of floating systems, and applications of these systems.
\end{abstract}

Key Words: Floating matrix, gastrointestinal, gastroretentive, system, evaluation, scintigraphy.

\section{INTRODUCTION}

The gastric emptying of dosage forms is an extremely variable process and ability to prolong and control the emptying time is a valuable asset for dosage forms that reside in the stomach for a longer period of time than conventional dosage forms. There are many difficulties faced in designing controlled release systems for better absorption and enhanced bioavailability. One of such difficulties is the inability to confine the dosage form in the desired area of the gastrointestinal tract. Drug absorption from the gastrointestinal tract is a complex procedure and is subject to many variables. It is widely acknowledged that the extent of gastrointestinal tract drug absorption is related to contact time with the small intestinal mucosa (Hirtz, 1985). Thus, small intestinal transit time is an important parameter for drugs that are incompletely absorbed. Basic human physiology with the details of gastric emptying, motility patterns, and physiological and formulation variables affecting the cosmic emptying are summarized. Gastroretentive systems can remain in the gastric region for several

\footnotetext{
*Corresponding Author:

Abhishek Chandel, Assistant Professor

Department of Pharmacy

Manav Bharti University

Solan -173229, Himachal Pradesh, India

E-mail: abhishek.k.chandel@gmail.com

Contact No.: 091-98177-10147
}

hours and hence significantly prolong the gastric residence time of drugs. Prolonged gastric retention improves bioavailability, reduces drug waste, and improves solubility for drugs that are less soluble in a high $\mathrm{pH}$ environment. It has applications also for local drug delivery to the stomach and proximal small intestines. Gastro retention helps to provide better availability of new products with new therapeutic possibilities and substantial benefits for patients. Based on these approaches, classification of floating drug delivery systems (FDDS) has been described in detail. In vivo/in vitro evaluation of FDDS has been discussed by scientists to assess the efficiency and application of such systems. Several recent examples have been reported showing the efficiency of such systems for drugs with bioavailability problems.

\section{Basic Gastrointestinal Tract Physiology}

Basically stomach is divided into 3 regions: fundus, body, and antrum (pylorus). The proximal part made of fundus and body acts as a reservoir for undigested material, the antrum is the main site for mixing motions and act as a pump for gastric emptying by propelling actions (Desai, 1984). Gastric emptying occurs during fasting as well as fed states. The pattern of motility is however distinct in the 2 states. During the fasting state an inter-digestive series of electrical events take place, which cycle both through stomach and intestine 
every 2 to 3 hours (Vantrappen et al., 1979). This is called the inter-digestive myloelectric cycle or migrating myloelectric cycle (MMC), which is further divided into following 4 phases as described by Wilson and Washington (Wilson and Washington, 1989)

Phase I (basal phase) lasts from 40 to 60 minutes with rare contractions.

Phase II (pre-burst phase) lasts for 40 to 60 minutes with intermittent action potential and contractions. As the phase progresses the intensity and frequency also increases gradually.

Phase III (burst phase) lasts for 4 to 6 minutes. It includes intense and regular contractions for short period. It is due to this wave that all the undigested material is swept out of the stomach down to the small intestine. It is also known as the housekeeper wave.

Phase IV lasts for 0 to 5 minutes and occurs between phases III and I of 2 consecutive cycles.

After the ingestion of a mixed meal, the pattern of contractions changes from fasted to that of fed state. This is also known as digestive motility pattern and comprises continuous contractions as in phase II of fasted state. These contractions result in reducing the size of food particles (to less than $1 \mathrm{~mm}$ ), which are propelled toward the pylorus in a suspension form. During the fed state onset of MMC is delayed resulting in slowdown of gastric emptying rate (Desai and Bolton, 1993). Scintigraphic studies determining gastric emptying rates revealed that orally administered controlled release dosage forms are subjected to basically 2 complications, that of short gastric residence time and unpredictable gastric emptying rate.

\section{CLASSIFICATION OF DRUG DELIVERY}

A. Single Unit Floating Dosage Systems

a) Effervescent Systems (Gas-generating Systems)

b) Non-effervescent Systems

B. Multiple Unit Floating Dosage Systems

a) Non-effervescent Systems

b) Effervescent Systems (Gas-generating Systems)

c) Hollow Microspheres

C. Raft Forming Systems

\section{A. Single Unit Floating Dosage Systems}

a) Effervescent Systems (Gas-generating Systems)
These buoyant systems utilized matrices prepared with swellable polymers like HPMC, polysaccharides like chitosan, effervescent components like sodium bicarbonate, citric acid and tartaric acid or chambers containing a liquid that gasifies at body temperature. The optimal stoichiometric ratio of citric acid and sodium bicarbonate for gas generation is reported to be $0.76: 1$. The common approach for preparing these systems involves resin beads loaded with bicarbonate and coated with ethylcellulose. The coating, which is insoluble but permeable, allows permeation of water. Thus, carbon dioxide is released, causing the beads to float in the stomach (Rubinstein and Friend, 1994). Excipients used most commonly in these systems include HPMC, polyacrylate polymers, polyvinyl acetate, Carbopol ${ }^{\circledR}$, agar, sodium alginate, calcium chloride, polyethylene oxide and polycarbonates

\section{b) Non-Effervescent Systems}

This type of system, after swallowing, swells unrestrained via imbibition of gastric fluid to an extent that it prevents their exit from the stomach. These systems may be referred to as the 'plug-type systems' since they have a tendency to remain lodged near the pyloric sphincter. One of the formulation methods of such dosage forms involves the mixing of drug with a gel, which swells in contact with gastric fluid after oral administration and maintains a relative integrity of shape and a bulk density of less than one within the outer gelatinous barrier. The air trapped by the swollen polymer confers buoyancy to these dosage forms. Examples of this type of FDDS include colloidal gelbarrier (Rubinstein and Friend, 1979), microporous compartment system (Roy, 1977), alginate beads (Whitehead et al., 1998), and hollow microspheres (Sato and Kawashima, 2003). Another type is a Fluid- filled floating chamber (Joseph et al., 2002) which includes incorporation of a gas-filled floatation chamber into a microporous component that houses a drug reservoir. Apertures or openings are present along the top and bottom walls through which the gastrointestinal tract fluid enters to dissolve the drug. The other two walls in contact with the fluid are sealed so that the undissolved drug remains therein. The fluid present could be air, under partial vacuum or any other suitable gas, liquid, or solid having an appropriate specific gravity and an inert behaviour. The device is of 


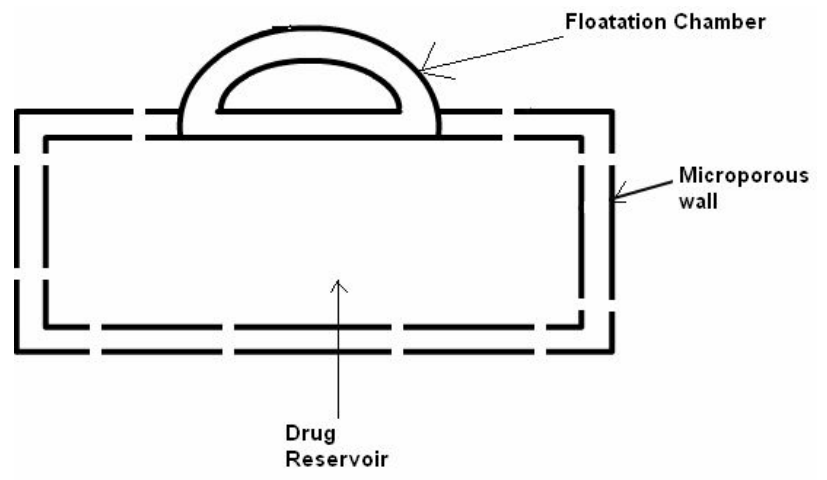

Figure 1: Gas filled floatation chamber

swallowable size, remains afloat within the stomach for a prolonged time and after the complete release the shell disintegrates, passes off to the intestine, and is eliminated.

A newer self-correcting floatable asymmetric configuration drug delivery system (Yang and Fassihi, 1996) has a 3-layermatrix to control the drug release. This 3-layer principle has been improved by development of an asymmetric configuration drug delivery system in order to modulate the release extent and achieve zero-order release kinetics by initially maintaining a constant area at the diffusing front with subsequent dissolution/erosion toward the completion of the release process. The system was designed in such a manner that it floated to prolong gastric residence time in vivo, resulting in longer total transit time within the gastrointestinal tract environment with maximum absorptive capacity and consequently greater bioavailability. This particular characteristic would be applicable to drugs that have $\mathrm{pH}$-dependent solubility, a narrow window of absorption, and are absorbed by active transport from either the proximal or distal portion of the small intestine.

\section{B. Multiple Unit Floating Systems}

In spite of extensive research and development in the area of HBS and other floating tablets, these systems suffer from an important drawback of high variability of gastrointestinal transit time, when orally administered, because of their all-or-nothing gastric emptying nature. In order to overcome the above problem, multiple unit floating systems were developed, which reduce the inter-subject variability in absorption and lower the probability of dosedumping. Reports have been found on the development of both non-effervescent and effervescent multiple unit systems (Iannuccelli et al., 1998). Much research has been focused and the scientists are still exploring the field of hollow microspheres, capable of floating on the gastric fluid and having improved gastric retention properties.

\section{a) Non-effervescent Systems}

No much report was found in the literature on noneffervescent multiple unit systems, as compared to the effervescent systems. However, few workers have reported the possibility of developing such system containing indomethacin, using chitosan as the polymeric excipient. A multiple unit HBS containing indomethacin as a model drug prepared by extrusion process is reported (Tardi and Troy, 2002). A mixture of drug, chitosan and acetic acid is extruded through a needle, and the extrudate is cut and dried. Chitosan hydrates and floats in the acidic media, and the required drug release could be obtained by modifying the drug-polymer ratio.

\section{b) Effervescent Systems (Gas-generating Systems)}

There are reports of sustained release floating granules containing tetracycline hydrochloride (Ikura et al., 1988). The granules are a mixture of drug granulates of two stages $\mathrm{A}$ and $\mathrm{B}$, of which $\mathrm{A}$ contains 60 parts of HPMC, 40 parts of polyacrylic acid and 20 parts of drug and B contains 70 parts of sodium bicarbonate and 30 parts of tartaric acid. 60 parts by weight of granules of stage A and 30 parts by weight of granules of stage $\mathrm{B}$ are mixed along with a lubricant and filled into capsule. In dissolution media, the capsule shell dissolves and liberates the granules, which showed a floating time of more than $8 \mathrm{~h}$ and sustained drug release of $80 \%$ in about6.5 h. Floating minicapsules of pepstatin having a diameter of 0.1-0.2 $\mathrm{mm}$ has been reported by Umezawa (Umezawa and Hamao, 1978). These minicapsules contain a central core and a coating. The central core consists of a granule composed of sodium bicarbonate, lactose and a binder, which is coated with HPMC. Pepstatin is coated on the top of the HPMC layer. The system floats because of the $\mathrm{CO}_{2}$ release in gastric fluid and the pepstatin resides in the stomach for prolonged period. Alginates have received much attention in the development of multiple unit systems. Alginates are non-toxic, biodegradable linear copolymers composed of Lglucuronic and L-mannuronic acid residues. A multiple unit system was prepared (Iannuccelli et 


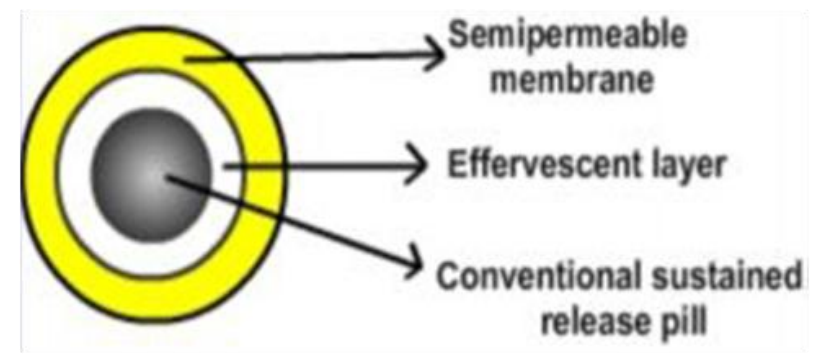

(a)

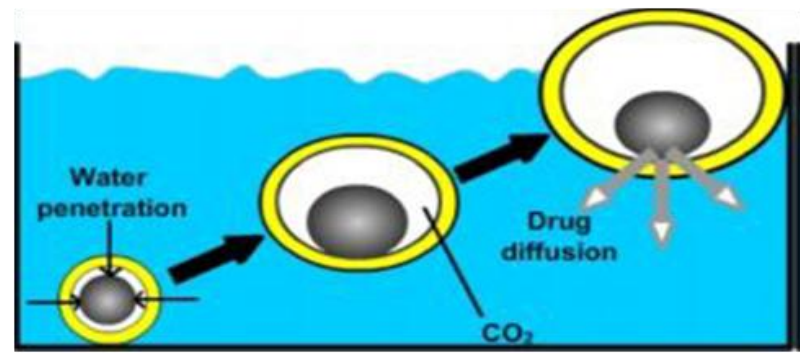

(b)

Figure 2: (a) Different layers-Semi permeable membrane, Effervescent Layer, Core pill layer, (b) Mechanism of floatation via $\mathrm{CO}_{2}$ generation.

al., 1998) comprises of calcium alginate core and calcium alginate/PVA membrane, both separated by an air compartment. In presence of water, the PVA leaches out and increases the membrane permeability, maintaining the integrity of the air compartment. Increase in molecular weight and concentration of PVA, resulted in enhancement of the floating properties of the system.

Freeze-drying technique is also reported for the preparation of floating calcium alginate beads (Stops et al., 2008). Sodium alginate solution is added drop wise into the aqueous solution of calcium chloride, causing the instant gelation of the droplet surface, due to the formation of calcium alginate. The obtained beads are freeze-dried resulting in a porous structure, which aid in floating. The authors studied the behaviour of radio labeled floating beads and compared with nonfloating beads in human volunteers using gamma scintigraphy. Prolonged gastric residence time of more than $5.5 \mathrm{~h}$ was observed for floating beads. The non-floating beads had a shorter residence time with a mean onset emptying time of $1 \mathrm{~h}$.

A new multiple type of floating dosage system had developed having a pill in the core, composed of effervescent layers and swellable membrane layers coated on sustained release pills (shown in figure 2). The inner layer of effervescent agents containing sodium bicarbonate and tartaric acid was divided into 2 sublayers to avoid direct contact between the 2 agents. These sublayers were surrounded by a swellable polymer membrane containing polyvinyl acetate and purified shellac. When this system was immersed in the buffer at $37^{\circ} \mathrm{C}$, it settled down and the solution permeated into the effervescent layer through the outer swellable membrane. $\mathrm{CO}_{2}$ was generated by the neutralization reaction between the 2 effervescent agents, producing swollen pills (like balloons) with a density less than $1.0 \mathrm{~g} / \mathrm{ml}$ (Ichikawa et al., 1991).

\section{c) Hollow Microspheres}

Hollow microspheres are considered as one of the most promising buoyant systems, as they possess the unique advantages of multiple unit systems as well as better floating properties, because of central hollow space inside the microsphere. The general techniques involved in their preparation include simple solvent evaporation and solvent diffusion and evaporation. The drug release and better floating properties mainly depend on the type of polymer, plasticizer and the solvents employed for the preparation. Polymers such as polycarbonate, Eudragit ${ }^{\circledR}$ Sand cellulose acetate were used in the preparation of hollow microspheres, and the drug release can be modulated by optimizing the polymer quantity and the polymer-plasticizer ratio. Sustained release floating microspheres using polycarbonate were developed (Thanoo et al., 1993), employing solvent evaporation technique. Aspirin, griseofulvin and p-nitroaniline were used as model drugs. Dispersed phase containing polycarbonate solution in dichloromethane, and micronized drug, was added to the dispersion medium containing sodium chloride, polyvinyl alcohol and methanol. The dispersion was stirred for 3-4h to assure the complete solvent evaporation, and the microspheres obtained were filtered, washed with coldwater and dried. The spherical and hollow nature of the microspheres was confirmed by Scanning electron microscopic studies. The microspheres showed a drug payload of more than $50 \%$, and the amount of 


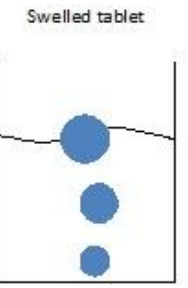

(a)

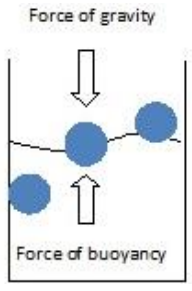

(b)

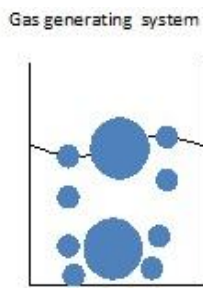

(c)
Figure 3: Different mechanisms of floating systems.

drug incorporated is found to influence the particle size distribution and drug release. The larger proportion of bigger particles was seen at high drug loading, which can be attributed to the increased viscosity of the dispersed phase.

\section{Raft Forming Systems}

Raft forming systems have received much attention for the delivery of antacids and drug delivery for gastrointestinal infections and disorders. The basic mechanism involved in the raft formation includes the formation of viscous cohesive gel in contact with gastric fluids, wherein each portion of the liquid swells forming a continuous layer called a raft. The raft floats because of the buoyancy created by the formation of $\mathrm{CO}_{2}$ and act as a barrier to prevent the reflux of gastric Contents like $\mathrm{HCl}$ and enzymes into the esophagus. Usually, the system contains a gel forming agent and alkaline bicarbonates or carbonates responsible for the formation of to make the system less dense and float on the gastric fluids (Paterson et al., 2008).

\section{MECHANISM OF FLOATING SYSTEMS}

There are various attempts have been made to retain the dosage form in the stomach as a way of increasing the retention time. These attempts include introducing floating dosage forms (gas-generating systems and swelling or expanding systems, mucoadhesive systems, high-density systems, modified shape systems, gastric-emptying delaying devices and co-administration of gastric-emptying delaying drugs. Among these, the floating dosage forms have been most commonly used. Floating drug delivery systems (FDDS) have a bulk density less than gastric fluids and so remain buoyant in the stomach without affecting the gastric emptying rate for a prolonged period of time. While the system is floating on the gastric contents (given in the Figure 3 (a)), the drug is released slowly at the desired rate from the system. After release of drug, the residual system is emptied from the stomach. This results in an increased GRT and a better control of the fluctuations in plasma drug concentration. However, besides a minimal gastric content needed to allow the proper achievement of the buoyancy retention principle, a minimal level of floating force (F) is also required to keep the dosage form reliably buoyant on the surface of the meal. To measure the floating force kinetics, a novel apparatus for determination of resultant weight has been reported in the literature. The apparatus operates by measuring continuously the force equivalent to $F$ (as a function of time) that is required to maintain the submerged object. The object floats better if $\mathrm{F}$ is on the higher positive side (Figure 3(b)). This apparatus helps in optimizing FDDS with respect to stability and durability of floating forces produced in order to prevent the drawbacks of unforeseeable intragastric buoyancy capability variations (Garg and Sharma, 2003).

$\mathrm{F}=\mathrm{F}$ buoyancy $-\mathrm{F}$ gravity $=(\mathrm{Df}-\mathrm{Ds}) \mathrm{gv}$

Where, $\mathrm{F}=$ total vertical force

$\mathrm{Df}=$ fluid density

Ds = object density

$\mathrm{v}=$ volume and

$\mathrm{g}=$ acceleration due to gravity

\section{ADVANTAGES OF FDDS SYSTEM}

(Babu and Khar, 1990; Kikani, 2001)

1. The gastroretentive systems are advantageous for drugs absorbed through the stomach, e.g. ferrous salts, antacids.

2. Acidic substances like aspirin cause irritation on the stomach wall when come in contact with it. Hence, HBS formulation may be useful for the administration of aspirin and other similar drugs.

3. Administration of prolongs release floating dosage forms, tablet or capsules, will result in dissolution of the drug in the gastric fluid. They dissolve in the gastric fluid would be available for absorption in the small intestine after emptying of the stomach contents. It is therefore expected that a drug will be fully absorbed from floating dosage forms if it remains in the solu- 
tion form even at the alkaline $\mathrm{pH}$ of the intestine.

4. The gastro retentive systems are advantageous for drugs meant for local action in the stomach. e.g. antacids.

5. When there is a vigorous intestinal movement and a short transit time as might occur in certain type of diarrhea, poor absorption is expected. Under such circumstances it may be advantageous to keep the drug in floating condition in stomach to get a relatively better response.

6. FDDS improves patient compliance by decreasing dosing frequency.

7. Bioavailability enhances despite first pass effect because fluctuations in plasma drug concentration are avoided; a desirable plasma drug concentration is maintained by continuous drug release.

8. Better therapeutic effect of short half-life drugs can be achieved.

9. Gastric retention time is increased because of buoyancy.

10. Enhanced absorption of drugs which solubilize only in stomach.

11. Superior to single unit floating dosage forms as such microspheres releases drug uniformly and there is no risk of dose dumping.

12. Avoidance of gastric irritation, because of sustained release effect, floatability and uniform release of drug through multi particulate system.

\section{EVALUATION PARAMETERS OF STOMACH SPECIFIC FDDS}

There are different studies reported in the literature indicate that pharmaceutical dosage forms exhibiting gastric residence in vitro floating behaviour show prolonged gastric residence in vivo. However, it has to be pointed out that good in vitro floating behaviour alone is not sufficient proof for efficient gastric retention in vivo. The effects of the simultaneous presence of food and of the complex motility of the stomach are difficult to estimate. Obviously, only in vivo studies can provide definite proof that prolonged gastric residence is obtained.

\section{Measurement of buoyancy capabilities of the FDDS}

The floating behaviour was evaluated with resultant weight measurements. The experiment was carried out in two different media, deionised water in order to monitor possible difference. The apparatus and its mechanism are explained earlier in this article. The results showed that higher molecular weight polymers with slower rate of hydration had enhanced floating behaviour and it was observed more in simulated meal medium compared to deionized water.

\section{Floating time and dissolution}

The test for floating time measurement is usually performed in stimulated gastric fluid or $0.1 \mathrm{~mole} / \mathrm{lit}$ $\mathrm{HCl}$ maintained at $37^{\circ} \mathrm{C}$. It is determined by using USP dissolution apparatus containing $900 \mathrm{ml}$ of $0.1 \mathrm{~mole} / \mathrm{lit} \mathrm{HCl}$ as the dissolution medium at $37^{\circ} \mathrm{C}$. The time taken by the dosage form to float is termed as floating lag time and the time for which the dosage form floats is termed as the floating or flotation time (Karande and Yeole, 2006)

A more relevant in-vitro dissolution method proposed to evaluate a floating drug delivery system (for tablet dosage form) (Gohel et al., 2004). A $100 \mathrm{ml}$ glass beaker was modified by adding a side arm at the bottom of the beaker so that the beaker can hold $70 \mathrm{ml}$ of $0.1 \mathrm{~mol} / \mathrm{lit} \mathrm{HCl}$ dissolution medium and allow collection of samples. A burette was mounted above the beaker to deliver the dissolution medium at a flow rate of $2 \mathrm{ml} / \mathrm{min}$ to mimic gastric acid secretion rate. The performance of the modified dissolution apparatus was compared with USP dissolution. Apparatus 2 (Paddle): The problem of adherence of the tablet to the shaft of the paddle was observed with the USP dissolution apparatus. The tablet did not stick to the agitating device in the proposed dissolution method. The drug release followed zero-order kinetics in the proposed method. Similarity of dissolution curves was observed between the USP method and the proposed method at $10 \%$ difference level (f2 $=57)$. The proposed test may show good in vitro-in vivo correlation since an attempt is made to mimic the in vivo conditions such as gastric volume, gastric emptying, and gastric acid secretion rate. 


\section{Drug release}

Dissolution tests are performed using the dissolution apparatus. Samples are withdrawn periodically from the dissolution medium with replacement and then analyzed for their drug content after an appropriate dilution.

\section{Content uniformity, hardness, friability (for tablets)}

5. Drug loading, drug entrapment efficiency, particle size analysis, surface characterization (for floating microspheres and beads)

Drug loading is assessed by crushing accurately weighed sample of beads or microspheres in a mortar and added to the appropriate dissolution medium which is then centrifuged, filtered and analyzed by various analytical methods like spectrophotometry. The percentage drug loading is calculated by dividing the amount of drug in the sample by the weight and simulated meal, total beads or microspheres. The particle size and the size distribution of beads or microspheres are determined in the dry state using the optical microscopy method. The external and cross-sectional morphology (surface characterization) is done by scanning electron microscope (SEM) (Agnihotri et al., 2006).

\section{X-Ray/Gamma scintigraphy}

$\mathrm{X}$-Ray/Gamma scintigraphy is a very popular evaluation parameter for floating dosage form now a day (Fell and Digenis, 1984). It helps to locate dosage form in the GIT and by which one can predict and correlate the gastric emptying time and the passage of dosage form in the GIT. Here the inclusion of a radio-opaque material into a solid dosage form enables it to be visualized by $\mathrm{X}$-rays. Similarly, the inclusion of a $\gamma$-emitting radionuclide in a formulation allows indirect external observation using a $\gamma$-camera or scinti-scanner (Harries and Sharma, 1990). In case of $\gamma$-scintigraphy, the $\gamma$-rays emitted by the radionuclide are focused on a camera, which helps to monitor the location of the dosage form in the GI tract (Timmermans et al., 1989).

\section{Pharmacokinetic studies}

Pharmacokinetic studies are the integral part of the in vivo studies and several works has been on that. The pharmacokinetics studies of verapamil, from the loading pellets containing drug, filled into a capsule, and compared with the conventional verapamil tablets of similar dose $(40 \mathrm{mg})$. The tmax and AUC (0-infinity) values $(3.75 \mathrm{~h}$ and 364.65 $\mathrm{ng} / \mathrm{mlh}$, respectively) for floating pellets were comparatively higher than those obtained for the conventional verapamil tablets (tmax value $1.21 \mathrm{~h}$, and AUC value $224.22 \mathrm{ng} / \mathrm{mlh}$ ) (Sawicki, 2002). No much difference was found between the Cmax values of both the formulations, suggesting the improved bioavailability of the floating pellets compared to the conventional tablets. An improvement in bioavailability has also been observed with piroxicam in hollow polycarbonate microspheres administered in rabbits. The microspheres showed about 1.4 times more bioavailability, and the elimination half-life was increased by about three times than the free drug.

\section{FACTORS CONTROLLING GASTRIC RETEN- TION OF DOSAGE FORMS}

The gastric retention time (GRT) of dosage forms is controlled by several factors such as density and size of the dosage form, food intake, nature of the food, posture, age, sex, sleep and disease state of the individual (e.g., gastrointestinal diseases and diabetes) and administration of drugs such as prokinetic agents (cisapride and metoclopramide).

\section{Density of dosage form}

Dosage forms having a density lower than that of gastric fluid experience floating behavior and hence gastric retention. A density of $<1.0 \mathrm{gm} / \mathrm{cm} 3$ is required to exhibit floating property. However, the floating tendency of the dosage form usually decreases as a function of time, as the dosage form gets immersed into the fluid, as a result of the development of hydrodynamic equilibrium (Timmermans and Moes, 1990).

\section{Size of dosage form}

The size of the dosage form is another factor that influences gastric retention. The mean gastric residence times of non-floating dosage forms are highly variable and greatly dependent on their size, which may be small, medium, and large units. In fed conditions, the smaller units get emptied from the stomach during the digestive phase and the larger units during the housekeeping waves. In most cases, the larger the size of the dosage form, 
the greater will be the gastric retention time because the larger size would not allow the dosage form to quickly pass through the pyloric antrum into the intestine (El-Kamel et al., 2001). Thus the size of the dosage form appears to be an important factor affecting gastric retention.

\section{Food intake and nature of food}

Food intakes, the nature of the food, caloric content, and frequency of feeding have a profound effect on the gastric retention of dosage forms. The presence or absence of food in the stomach influences the GRT of the dosage form. Usually, the presence of food increases the GRT of the dosage form and increases drug absorption by allowing it to stay at the absorption site for a longer time. In a gamma scintigraphic study of a bilayer floating capsule of misoprostol (Oth et al., 1992), the mean gastric residence time was $199 \pm 69$ minutes; after a light breakfast, a remarkable enhancement of average GRT to $618 \pm 208$ minutes was observed.

\section{Effect of gender, posture and age}

A study (Mojaverian et al., 1988) found that females showed comparatively shorter mean ambulatory GRT than males, and the gastric emptying in women was slower than in men. The authors also studied the effect of posture on GRT, and found no significant difference in the mean GRT for individuals in upright, ambulatory and supine state. On the other hand, in a comparative study in humans, the floating and non-floating systems behaved differently (Gansbeke et al., 1991). In the upright position, the floating systems floated to the top of the gastric contents and remained for a longer time, showing prolonged GRT. But the non-floating units settled to the lower part of the stomach and underwent faster emptying as a result of peristaltic contractions, and the floating units remained away from the pylorus. However, in supine position, the floating units are emptied faster than non-floating units of similar size (Timmermans and Moes, 1994).

\section{APPLICATION OF FDDS}

Floating drug delivery offers several applications for drugs having poor bioavailability because of the narrow absorption window in the upper part of the gastrointestinal tract. It retains the dosage form at the site of absorption and thus enhances the bioavailability. These are summarized as follows:

\section{Sustained Drug Delivery}

HBS systems can remain in the stomach for long periods and hence can release the drug over a prolonged period of time. The problem of short gastric residence time encountered with an oral $C R$ formulation hence can be overcome with these systems. These systems have a bulk density of $<1$ as a result of which they can float on the gastric contents. These systems are relatively large in size and passing from the pyloric opening is prohibited.

\section{Site-Specific Drug Delivery}

These systems are particularly advantageous for drugs that are specifically absorbed from stomach or the proximal part of the small intestine, e.g., riboflavin and furosemide. Furosemide is primarily absorbed from the stomach followed by the duodenum. It has been reported that a monolithic floating dosage form with prolonged gastric residence time was developed and the bioavailability was increased. AUC obtained with the floating tablets was approximately 1.8 times those of conventional furosemide tablets.

\section{Absorption Enhancement}

Drugs that have poor bioavailability because of site specific absorption from the upper part of the gastrointestinal tract are potential candidates to be formulated as floating drug delivery systems, thereby maximizing their absorption. E.g. A significantly increase in the bioavailability of floating dosage forms (42.9\%) could be achieved as compared with commercially available LASIX tablets $(33.4 \%)$ and enteric coated LASIX-long product (29.5\%) (Moursy et al., 2003).

\section{CONCLUSION}

The FDDS become an additional advantage for drugs that are absorbed primarily in the upper part of GI tract, i.e., the stomach, duodenum, and jejunum. Drug absorption in the gastrointestinal tract is a highly variable procedure and prolonging gastric retention of the dosage form extends the time for drug absorption. FDDS promises to be a potential approach for gastric retention. It seems that to formulate an efficient FDDS is sort of a challenge and the work will go on and on until an ideal approach with industrial applicability and feasibility arrives. 


\section{REFERENCES}

Agnihotri, S.A., Jawalkar, S.S., Aminabhavi, T.M. (2006). Controlled release of cephalexin through gellan gum beads: Effect of formulation parameters on entrapment efficiency, size, and drug release, Eur. J. Pharm. Biopharm. 63:249-261. DOI PMid:16621483

Babu, V.B.M., Khar, R.K. (1990). In vitro and In vivo studies of sustained release floating dosage forms containing salbutamol sulphate. Pharmazie.; 45: 268-270. PMid:2381979

Desai, S., Bolton, S. (1993). A floating controlled release drug delivery system: in vitro- in vivo evaluation. Pharm Res; 10(9): 1321-1325. DOI PMid:8234170

Desai S. (1984) A Novel Floating Controlled Release Drug Delivery System Based on a Dried Gel Matrix Network [master's thesis]. [thesis]. Jamaica, NY: St John's University.

El-Kamel, A.H., Sokar, M.S., Al Gamal, S.S., Naggar, V.F. (2001) Preparation and evaluation of ketoprofen floating oral delivery system, Int J Pharm. 220(1-2): 13-21. DOI PMid: 11376963

Fell, J., Digenis, C.G. (1984) Imaging and behavior of solid oral dosage forms in vivo. Int. J. Pharm. 22(1): 1-15. DOI

Gansbeke BV, Timmermans J, Schoutens A, Moes AJ.(1991) Intragastric positioning of two concurrently ingested pharmaceutical matrix dosage forms, Nucl Med Biol. 18: 711-718.

Garg S. and Sharma S. (2003). Gastroretentive Drug Delivery System, Business Briefing: Pharmatech., 160-166

Gohel M.C., Mehta P.R., Dave, R.K., Bariya, N.H. (2004). A More Relevant Dissolution Method for Evaluation of Floating Drug Delivery System, Dissolutiontech. 11(4): 22-25.

Harries, D., Sharma, H.L. (1990) GI transit of potential bioadhesive formulations in man: Ascintigraphic study, J. Cont. Rel., 12(1): 4553. DOI

Kikani, H.N. (2001). A Thesis on, Floating Drug Delivery System, The North Gujarat University, Patan, 11-12.

Hirtz, J. (1985) The gastrointestinal absorption of drugs in man: a review of current concepts and methods of investigation. Br J Clin Pharmacol., 19: 77S-83S. PMid:3890912 PMCid:1463764

Iannuccelli, V., Coppi, G., Sansone, R., Ferolla, G. (1998). Air compartment multiple-unit system for prolonged gastric residence. Part II. In-vivo evaluation. Int. J. Pharm. 174:55-62. DOI

Iannuccelli, V., Coppi, G., Bernabei, M.T., Cameroni, R. (1998) Air compartment multiple-unit system for prolonged gastric residence. Part I. Formulation study, Int.J.Pharm.174: 47-54. DOI

Ichikawa, M., Watanabe, S., Miyake, Y. (1991). A new multiple unit oral floating dosage system. I: Preparation and in vitro evaluation of floating and sustained-release kinetics, J. Pharm. Sci. 80: 10621066. DOI PMid: 1815057

Ikura, Hiroshi, Suzuki, Yoshiki. (1988)United States Patent 4777033.

Joseph, N.H., Laxmi, S., Jayakrishnan, A. (2002). A floating type oral dosage form for piroxicam based on hollow microspheres: in vitro and invivo evaluation in rabbits, J. Cont. Rel., 79: 71-79. DOI

Karande, A.D., Yeole, P.G. (2006). Comparative Assessment of Different Dissolution Apparatus for Floating Drug Delivery Systems. Dissolutiontech. 13(1): 20-23.

Mojaverian, P., Vlasses, P.H., Kellner, P.E., Rocci, M.L. Jr. (1988) Effects of gender, posture, and age on gastric residence time of an indigestible solid: pharmaceutical considerations, Pharm Res. 10: 639-644. DOI PMid:3244616
Moursy, N.M., Afifi, N.H., Ghorab, D.M., El-Saharty, Y. (2003). Formulation and evaluation of sustained release floating capsules of Nicardipine hydrochloride, Pharmazie, 58: 38-43. PMid:12622251

Oth, M., Franz, M., Timmermans, J., Moes, A., (1992). The bilayer floating capsule: a stomach-directed drug delivery system for misoprostol, Pharm Res, 9: 298-302. DOI PMid:1614959

Paterson, R.S., Foster, J.E., O'Mahony, B., Stevens, H.N.E., Eccleston, G.M., Murray, J.G. (2000). An assessment of floating raft formation in man using magnetic resonance imaging (MRI). J Pharm Pharmacol., 8: S2 (suppl)

Roy H.M, U.S. (1977). Patent no. 4055178.

Rubinstein A., Friend D.R. (1994) Specific delivery to the gastrointestinal tract, in: Domb A. J (Ed.), Polymeric Site-Specific Pharmacotherapy, Wiley, Chichester, 282-283.

Rubinstein A., Friend D.R. (1979). U.S. Patent no. 4140755.

Sato, Y., Kawashima, Y. (2003). Physicochemical properties to determine the buoyancy of hollow microspheres (microballoons) prepared by the emulsion solvent diffusion method, Eur. J. Pharm. Sci., 55: 297-304

Sawicki, W. (2002). Pharmacokinetics of verapamil and norverapamil from controlled release floating pellets in humans. Eur. J. Pharm. Biopharm. 53: 29-35. DOI

Stops, F., Fell, J.T., Collett, J.H., Martini, L.G. (2008). Floating dosage forms to prolong gastroretention--the characterisation of calcium alginate beads, Int. J. Pharm., 350: 301-311. DOI PMid:17964096

Tardi, P., Troy, H. (2002) European patent no.EP1432402.

Thanoo, B.C, Sunny, M.C., Jayakrishnan, A. (1993). Oral sustainedrelease drug delivery systems using polycarbonate microspheres capable of floating on the gastric fluid, J. Pharm. Pharmacol., 45: 21-24. DOI PMid:8094440

Timmermans, J., Moes, A.J. (1990). How well do floating dosage forms float?, Int J Pharm. 62: 207-216. DOI

Timmermans, J., Moes AJ. (1994) Factors controlling the buoyancy and gastric retention capabilities of floating matrix capsules: new data for reconsidering the controversy, J Pharm Sci, 83(1): 18-24. DOI PMid:8138903

Timmermans, J.,Gansbeke, V.B., Moes, A.J. (1989). Assessing by gamma scintigraphy the in vivo buoyancy of dosage forms having known sizeand floating force profiles as a function of time. Vol I. Proceedings of the $5^{\text {th }}$ International Conference on Pharmacy Technology, Paris, France: APGI, 42-51.

Umezawa, Hamao.(1978) United States Patent no. 4101650.

Vantrappen, G.R., Peeters, T.L., Janssens, J. (1979). The secretory component of interdigestivemigratory motor complex in man. Scand J Gastroenterol. 14: 663-667. DOI PMid:93773

Whitehead, L., Fell, J., Sharma, H.L. (1998) Floating dosage forms: an in vivo study demonstrating prolonged gastric retention, J. cont. Rel. 55: 3-12. DOI

Wilson, C.G., Washington, N. (1989) The stomach: its role in oral drug delivery. In: Rubinstein MH, ed. Physiological Pharmaceutical: Biological Barriers to Drug Absorption. Chichester, UK: Ellis Horwood. Pp. 47-70.

Yang, L., Fassihi, R. (1996) Zero order release kinetics from selfcorrecting floatable configuration drug delivery system. J. Pharm. Sci., 85: 170-173. DOI PMid:8683443 